\title{
BRMS1 participates in regulating cell sensitivity to DNA interstrand crosslink damage by interacting with FANCI
}

\author{
JIANMING DOU*, YIREN ZHOU* ${ }^{*}$ XUNI LIU, XIAOJING QIAO, XI YANG, \\ WENJUAN XIE, SHOUYI QIAO and YANHUA WU \\ Institute of Genetics, School of Life Sciences, Fudan University, Shanghai 200433, P.R. China
}

Received May 6, 2018; Accepted September 27, 2018

DOI: $10.3892 /$ or.2018.6816

\begin{abstract}
Breast cancer metastasis suppressor 1 (BRMS1) is a tumor metastasis suppressor implicated in multiple steps during the metastatic cascade. Many proteins interacting with BRMS1 have been identified to unravel the intracellular signaling mechanisms. In the present study, we report that FANCI is a novel interacting protein of BRMS1 as determined by co-immunoprecipitation assay. The linker region between two coiled-coil motifs of BRMS1 is required for BRMS1-FANCI interaction. FANCI is an essential protein in the Fanconi anemia (FA) pathway responsible for the repair of DNA interstrand crosslinks (ICLs). We demonstrated that knockdown or knockout of BRMS1 significantly diminished the monoubiquitination of FANCI and FANCD2 in response to DNA ICL damage. BRMS1-deficient cells exhibited suppressed FANCD2 foci formation and hypersensitivity to ICLs. Moreover, rescue assays by utilizing different BRMS1 constructs suggested that BRMS1-FANCI interaction is necessary for the regulatory role of BRMS1 in the FA pathway. Overall, our findings characterize BRMS1 as a novel regulatory protein functioning in the DNA repair pathway via protein interaction.
\end{abstract}

\section{Introduction}

Breast cancer metastasis suppressor 1 (BRMS1) was first discovered for its significant inhibition of metastasis in breast cancer cells (1). Depending on the cell types used, BRMS1 has been noted to inhibit multiple steps in the invasion-metastasis cascade, including cell communication $(2,3)$, cell

Correspondence to: Professor Yanhua Wu, Institute of Genetics, School of Life Sciences, Fudan University, Shanghai 200433, P.R. China E-mail: yanhuawu@fudan.edu.cn

*Contributed equally

Key words: breast cancer metastasis suppressor 1, BRMS1, interstrand crosslinks, Fanconi anemia, protein interaction, ubiquitination migration (4,5), cell apoptosis $(6,7)$, epithelial-mesenchymal transition (EMT) (8), among others. Several important BRMS1-interacting proteins have been identified, providing possible clues to the molecular mechanisms of action of BRMS1. For instance, BRMS1 participates in histone modification and transcriptional regulation through interaction with the mSin3·HDAC complex (9). BRMS1 also interacts with the RelA/p65 subunit of NF- $\kappa \mathrm{B}$ and promotes binding of HDAC1 to RelA/p65, which suppresses the transcriptional activity of NF- $\kappa \mathrm{B}$ (6). More recently, BRMS1 has been found to be posttranslationally regulated by $\mathrm{CK} 2 \alpha$ via protein interaction, which affects the nuclear exportation and degradation of BRMS1 (10). Structural mapping reveals that two coiled-coil motifs and the internal linker region may be important for the different protein interactions of BRMS1 $(9,11,12)$.

Fanconi anemia (FA) is a rare autosomal or X-linked recessive inherited disease first described by Dr Guido Fanconi in 1927 (13). Although FA patients mainly suffer from bone marrow failure, many of them also display profound genome instability correlating with cancer predisposition (14). A higher risk of head and neck squamous cell carcinoma, leukemia, vulvar carcinoma, breast and ovarian cancer has been described in different FA patients (15). On the molecular level, the FA pathway plays a role in resolving DNA damage, especially interstrand crosslinks (ICLs) which covalently link the double strands of the DNA. Removal of ICLs is particularly important for cellular development, as ICLs strongly affect molecular processes which require DNA unwinding and strand separation such as DNA replication as well as transcription (16).

To date, 21 FA genes including FANCI have been identified. FANCI was first characterized in 2007 by Smogorzewska as a paralog of another FA gene, FANCD2. In response to DNA damage, FANCI binds to FANCD2 to form a heterodimeric FANCI-FANCD2 (FANCI/D2) complex. The FANCI/D2 complex is then monoubiquitinated, and downstream DNA repair proteins are further recruited to ICL sites (17). Afterwards, ICLs are removed so that genome stability can be guarded and cells can survive from DNA damage. As an essential component of the FANCI/D2 complex, FANCI is not only required for the stability of FANCD2 (18), but is also required for efficient FA core complex foci formation (19). 
In the present study, we revealed an interactive relationship between FANCI and BRMS1 through co-immunoprecipitation assay for the first time. The association relationship prompted us to ascertain whether BRMS1 has a role in the regulation of cell sensitivity to DNA damage. Our results showed that depletion of BRMS1 significantly diminished the monoubiquitination of FANCI and FANCD2, leading to a reduced FANCD2 foci formation and cell viability in response to ICL damage.

\section{Materials and methods}

Cell culture and transfection. 293T and U2OS cells were purchased from the Cell Bank of the Chinese Academy of Sciences (Shanghai, China). All cells were cultured in Dulbecco's modified Eagle's medium (DMEM) supplemented with $10 \%$ fetal bovine serum (FBS) in a $5 \% \mathrm{CO}_{2}$-humidified atmosphere at $37^{\circ} \mathrm{C}$. All the cell culture reagents were purchased from Gibco/Thermo Fisher Scientific, Inc. (Waltham, MA, USA). Cells at $70 \%$ confluency were transfected with Invitrogen $^{\mathrm{TM}}$ Lipofectamine 3000 (Thermo Fisher Scientific, Inc.) according to the manufacturer's instructions. In experiments evaluating cell sensitivity to ICL damage, mitomycin $\mathrm{C}$ (MMC; Roche Diagnostics, Indianapolis, IN, USA) was added to the cell culture medium at the indicated dosages.

Immunoprecipitation. Cells were harvested with cell lysis buffer (Thermo Fisher Scientific, Inc.) supplemented with protease inhibitor cocktail (Thermo Fisher Scientific, Inc.) and the lysate was centrifuged at $12,000 \mathrm{x} \mathrm{g}$ at $4^{\circ} \mathrm{C}$ for $15 \mathrm{~min}$. The supernatant was precleared with protein $\mathrm{A} / \mathrm{G}$ beads (Thermo Fisher Scientific, Inc.) and incubated with $1 \mu \mathrm{g}$ specific primary antibodies at $4^{\circ} \mathrm{C}$ overnight. The related antibodies included anti-Myc (cat. no. 05-724; Millipore, Bedford, MA, USA), anti-Flag (cat. no. F3165; Sigma-Aldrich; Merck KGaA, Darmstadt, Germany) and anti-FANCI (cat. no. ab15344; Abcam, Cambridge, MA, USA). Afterwards, protein A/G beads were added into the mixture and incubated at $4^{\circ} \mathrm{C}$ for at least $2 \mathrm{~h}$. After washing four times, the beads were resuspended in loading buffer and stored at $-20^{\circ} \mathrm{C}$ before being subjected to western blot analysis. Related recombinant plasmids used in the co-immunoprecipitation include Myc-BRMS1, BRMS1 deletion mutants as previously described (20) and Flag-FANCI (a kind gift from Professor Jun Huang of Zhejiang University, China).

Western blot analysis. Protein samples were collected with SDS lysis buffer. Protein samples (15-30 $\mu \mathrm{g})$ were separated by SDS-PAGE with 6 or $12 \%$ gel depending on specific experiment and then transferred into PVDF membranes. After blocking in $5 \%$ fat-free milk for $1 \mathrm{~h}$, the membranes were incubated with specific primary antibodies at $4^{\circ} \mathrm{C}$ overnight. Afterwards, the membranes were washed and incubated with secondary antibodies at room temperature for $1 \mathrm{~h}$. Membranes were visualized by enhanced chemiluminescence (ECL) kit (GE Healthcare Life Sciences, Logan, UT, USA). The images are representatives of several independent experiments with consistent results and the densitometric values were quantified with Gene Tools from Syngene software (Frederick, MD, USA). The related antibodies included anti-Myc (1:3,000 dilution; cat. no. 05-724; Millipore), anti-Flag (1:3,000 dilution;
Sigma-Aldrich), anti-FANCI (1:2,000 dilution; cat. no. ab15344; Abcam), anti-BRMS1 (1:3,000 dilution; cat. no. 16096-1-AP; Proteintech Group, Wuhan, China), anti-FANCD2 (1:2,000 dilution; cat. no. ab2187; Abcam) and peroxidase-conjugated goat anti-mouse (cat. no. IH-0031; DingGuo Bio., Beijing, China)/rabbit (cat. no. IH-0011; DingGuo Bio.) IgG diluted at $1: 3,000$ with $1 \%$ fat-free milk

Quantitative real-time PCR ( $q R T-P C R)$. Total RNA was extracted from cultured cells using Invitrogen ${ }^{\mathrm{TM}}$ Trizol (Thermo Fisher Scientific, Inc.) and 500 ng RNA was applied for reverse transcription using reverse transcriptase (Takara Biotechnology Co., Ltd., Dalian, China). Quantitative real-time PCR analysis was performed using SYBR-Green Supermix kit (Takara Biotechnology Co., Ltd.) with the CFX Connection detection system (Bio-Rad Laboratories, Inc., Hercules, CA, USA). Reactions with no cDNA template were performed as negative controls to rule out contamination. Primers for BRMS1 and internal control were previously described (7). Data were analyzed as previously described (21).

Plasmid construction and selection of stable transfectants. Recombinant pLenti-BRMS1-sgRNA plasmid was previously described (20). U2OS cells were transfected with pLenti-BRMS1-sgRNA or empty vector before being subjected to selection with puromycin. BRMSI-deficient clones and the control clones were selected through anti-BRMS1 immunoblotting. Genomic DNA of these clones was isolated to confirm BRMS1 mutation via Sanger sequencing.

Immunofluorescence. U2OS cells were cultured in a 24-well plate with coverslips inside and further treated with $1 \mu \mathrm{M}$ MMC for $24 \mathrm{~h}$. Cells grown on coverslips were then washed, fixed with $4 \%$ paraformaldehyde for $15 \mathrm{~min}$ and then permeabilized with $0.2 \%$ Triton for $15 \mathrm{~min}$. Cells were blocked in 5\% BSA supplemented with $5 \%$ donkey serum for $1 \mathrm{~h}$, and then incubated with primary antibodies in a wet container at $4^{\circ} \mathrm{C}$ overnight, followed by incubation with the fluorescence-conjugated secondary antibodies at $37^{\circ} \mathrm{C}$ for 45 min. Finally, cells were counterstained with Hoechst 33258 (Sigma-Aldrich) at $37^{\circ} \mathrm{C}$ for $20 \mathrm{~min}$. The fluorescence images were captured using an Axio Observer Z1 microscope (Carl Zeiss, Oberkochen, Germany). Antibodies used in the immunofluorescence included anti-FANCD2 (1:600 dilution; cat. no. ab2187; Abcam), anti-Myc (1:200 dilution; cat. no. 05-724; Millipore), Alexa Fluor 488 goat anti-mouse IgG (1:500 dilution; cat. no. A-21202; Thermo Fisher Scientific, Inc.) and Alexa 555 goat anti-rabbit IgG (1:300 dilution; cat. no. A-21430; Thermo Fisher Scientific, Inc.).

MMC sensitivity assay. Cells were seeded in 96-well plates at a density of 1,200 cells/well. After treatment with different concentrations of MMC for 3 days, cell viability was calculated with MTS assay (Promega, Madison, WI, USA) according to the manufacturer's instructions. OD values were scanned by a microplate reader (BioTek Instruments, Inc., Winooski, VT, USA).

Statistical analysis. Comparisons of quantitative data were analyzed by Student's t-test. We considered two groups with 
A

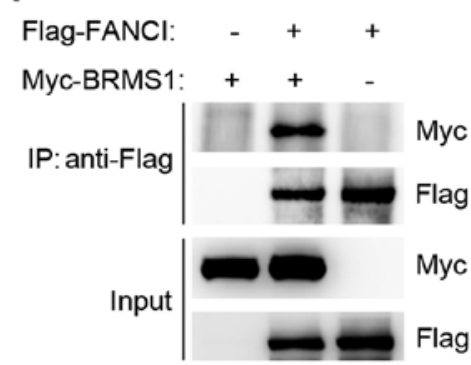

D

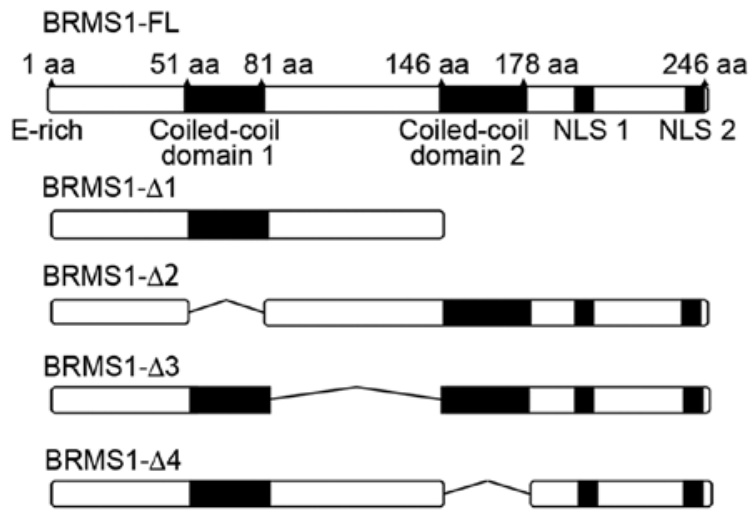

B

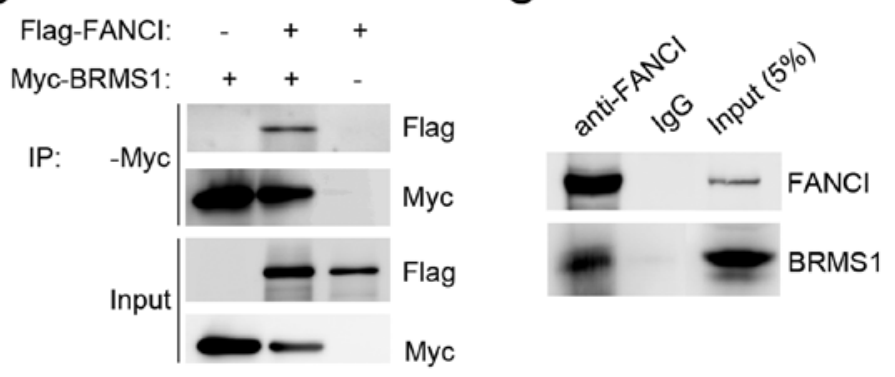

E

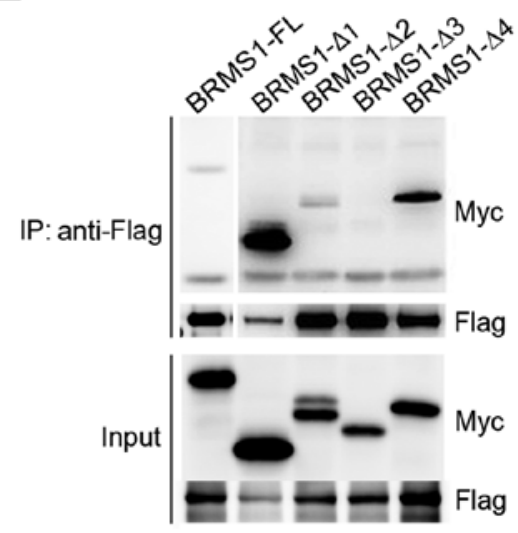

Figure 1. BRMS1 interacts with FANCI in vivo. (A and B) 293 T cells were transfected with indicated plasmids. Cell lysates were prepared and subjected to immunoprecipitation with the (A) anti-Flag or (B) anti-Myc antibody. The immunoprecipitates were detected with anti-Flag and anti-Myc antibodies. (C) U2OS cell lysates were prepared and subjected to immunoprecipitation with the anti-FANCI antibody. The immunoprecipitates were detected with anti-BRMS1 and anti-FANCI antibodies. IgG was utilized as a negative control. Five percent of the input was applied in parallel. (D) Schematic representations of BRMS1-FL and BRMS1 deletion mutants. (E) Different Myc-tagged BRMS1 plasmids were separately co-transfected with Flag-FANCI into 293T cells. Cell lysates were prepared and subjected to immunoprecipitation with the anti-Myc antibody. The immunoprecipitates were detected with anti-Myc and anti-Flag antibodies. BRMS1, breast cancer metastasis suppressor 1.

a P-value $<0.05$ to be different, and with a P-value $<0.01$ to be significantly different (labeled with ${ }^{*}$ and ${ }^{* *}$, respectively, in the figures).

\section{Results}

BRMS1 interacts with FANCI through its linker region. Previously, we reported that BRMS1 is able to interact with DBC1 through a large-scale tandem affinity purification (20). In the present study, another potential BRMS1-interacting protein, FANCI, was confirmed by co-immunoprecipitation experiment. As shown in Fig. 1A and B, when Myc-tagged BRMS1 and Flag-tagged FANCI were co-expressed in 293T cells, Flag-FANCI was detected in anti-Myc immunoprecipitates and vice versa. No binding was detected in the control cells. In addition, U2OS cells were utilized to detect the association between endogenous FANCI and BRMS1. As shown in Fig. 1C, BRMS1 was readily immunoprecipitated with anti-FANCI antibody, but not with the IgG control. These data strongly suggest that BRMS1 is able to interact with FANCI.

To identify the region of BRMS1 which is responsible for BRMS1-FANCI interaction, a series of deletion mutants of BRMS1 were utilized as previously described (Fig. 1D) (20). As shown in Fig. 1E, among all the mutants, only BRMS1- $\Delta 3$, which lost the linker region between two coiled-coil motifs (residues 81-146) abolished the binding ability of
BRMS1 with FANCI. By contrast, loss of either coiled-coil region (BRMS1- $\Delta 2$, BRMS1- $\Delta 4$ ) or the C-terminal domain (BRMS1- $\Delta 1$ ) had no effect on BRMS1-FANCI interaction.

Knockdown of BRMS1 decreases the monoubiquitination of FANCI/D2 upon DNA damage. An effective siRNA targeting BRMS1 was utilized as previously described (7), and it successfully suppressed endogenous BRMS1 expression in U2OS cells (siBRMS1) by comparison with U2OS control cells (siCtrl) at both the mRNA and protein level (Fig. 2A). MMC is widely used for inducing DNA ICLs. As shown in Fig. 2B, although both siBRMS1 and siCtrl cells exhibited monoubiquitiniation of FANCI/FANCD2 with MMC treatment, knockdown of BRMS1 led to a significant reduction in FANCI/D2 monoubiquitination levels. Statistical analysis revealed that the relative quantity of monoubiquitinated FANCI and FANCD2 declined by 52 and $28 \%$ separately (Fig. 2C and D). These data initially indicated a potential role of BRMS1 in regulating FANCI/D2 monoubiquitination in the response to MMC-induced ICLs.

BRMS1 is involved in FANCD2 foci formation upon DNA damage. When FANCI/D2 are monoubiquitinated, they gather to the damage sites in the nucleus and further recruit downstream exonucleases to repair DNA, leading to FANCD2 foci formation. To answer whether BRMS1 is also involved 
A

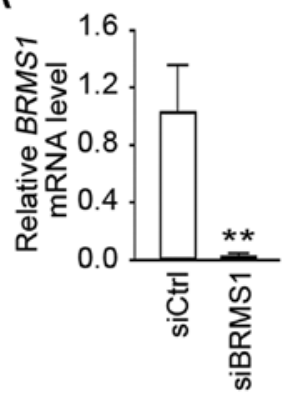

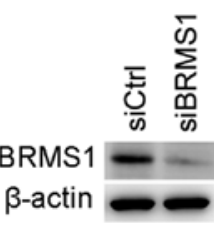

C

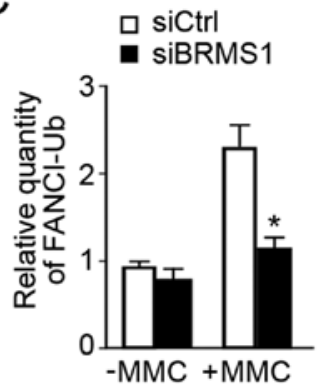

B

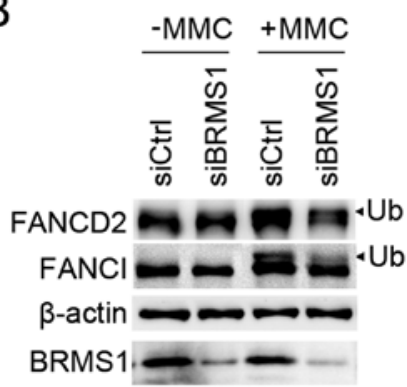

D

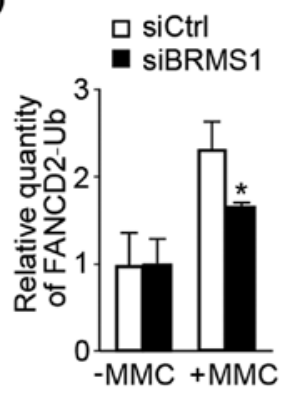

Figure 2. Knockdown of BRMS1 affects the monoubiquitination of FANCI and FANCD2 with MMC treatment. (A) Western blot (right panel) and qRT-PCR analysis (left panel) of U2OS cells transfected with the indicated siRNAs. $\beta$-actin was used as a loading control in the western blotting. Relative BRMS1 mRNA level was normalized to the internal control $18 \mathrm{~S}$ rRNA and expressed as mean $\pm \mathrm{SD}, \mathrm{n}=3$. (B) Immunoblot analysis of FANCI and FANCD2 in siBRMS1 and siCtrl cells with or without MMC $(120 \mathrm{nM})$ treatment for $24 \mathrm{~h}$. The monoubiquitinated forms of FANCI and FANCD2 are indicated by arrows. The relative quantity of monoubiquitinated (C) FANCI and (D) FANCD2 in siBRMS1 cells were normalized to that of the untreated siCtrl cells and are expressed as mean $\pm \mathrm{SD}, \mathrm{n}=3$; $\mathrm{P}<0.05$ and ${ }^{* *} \mathrm{P}<0.01$, compared to siCtrl cells. BRMS1, breast cancer metastasis suppressor 1; MMC, mitomycin C.

A
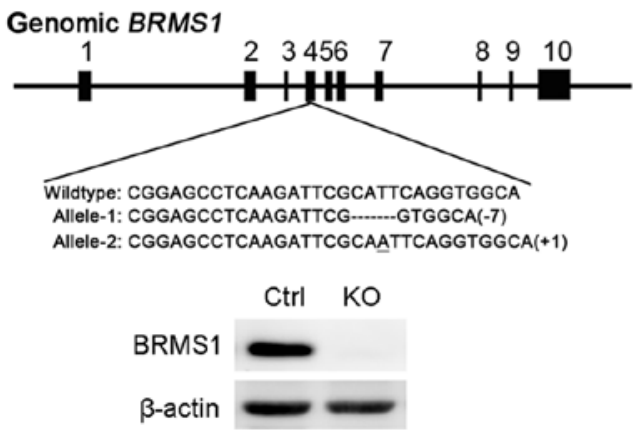

C

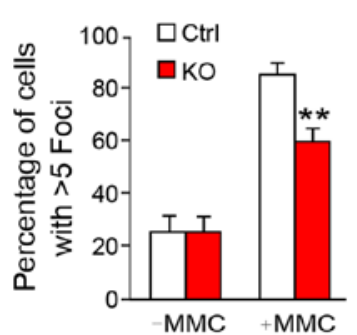

B

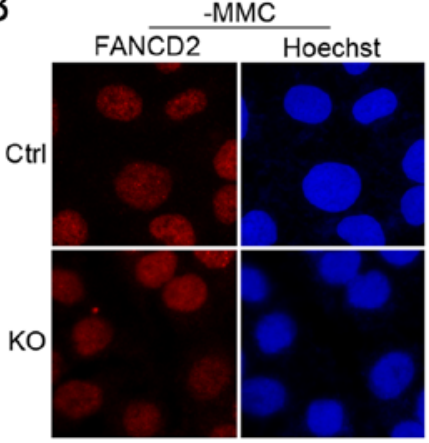

E
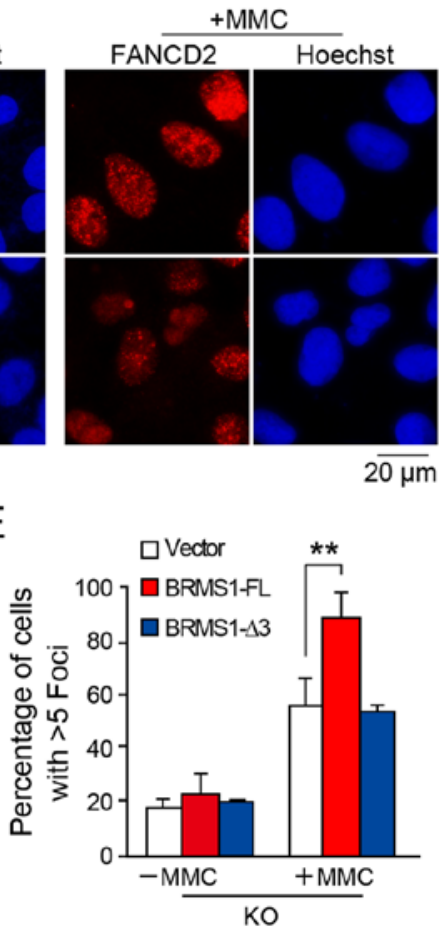

Figure 3. BRMS1-FANCI interaction is required for FANCD2 localization to ICL damage sites. (A) A schematic of sgRNA targeting the fourth exon of the $B R M S 1$ gene (upper panel). Different mutant alleles were identified in the BRMS1-knockout (KO) clone. Deletion is indicated by a dashed line and the insertion is underlined. The numbers of altered basepairs are shown in the brackets following the sequences. Western blot analysis of BRMS1 in U2OS clones is shown in the bottom panel. $\beta$-actin was used as a loading control. (B) Anti-FANCD2 immunofluorescence staining was performed in KO and Ctrl clones with or without MMC treatment $(1 \mu \mathrm{M})$ and cells were counterstained with Hoechst 33258. Representative fluorescence images of FANCD2 foci are shown. Scale bar, $20 \mu \mathrm{m}$. (C) Quantification analysis of the percentages of cells with $>5$ FANCD2 foci inside the nucleus in the KO/Ctrl clones. Values are presented as mean \pm SD, $\mathrm{n}=30$; ${ }^{* *} \mathrm{P}<0.01$, compared to the Ctrl. (D) Representative fluorescence images of KO cells reconstituted with Myc-tagged BRMS1-FL or BRMS1- $\Delta 3$ with MMC treatment. Scale bar, $20 \mu \mathrm{m}$. (E) Quantification analysis of the percentages of cells with $>5$ FANCD2 foci inside the nucleus in KO clones reconstituted with indicated plasmids. Values are presented as mean $\pm \mathrm{SD}, \mathrm{n}=30 ;{ }^{* *} \mathrm{P}<0.01$. BRMS1, breast cancer metastasis suppressor 1 ; MMC, mitomycin C; ICLs, interstrand crosslinks. 
A

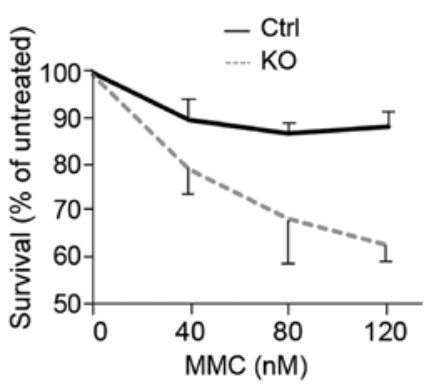

B

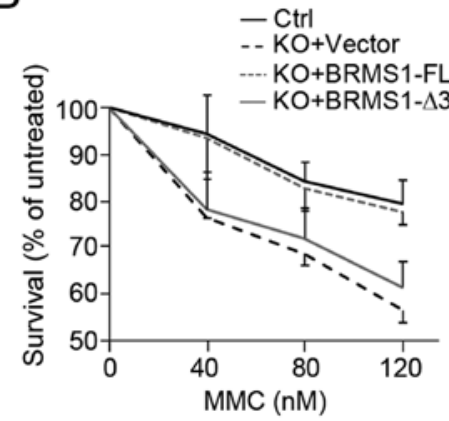

C

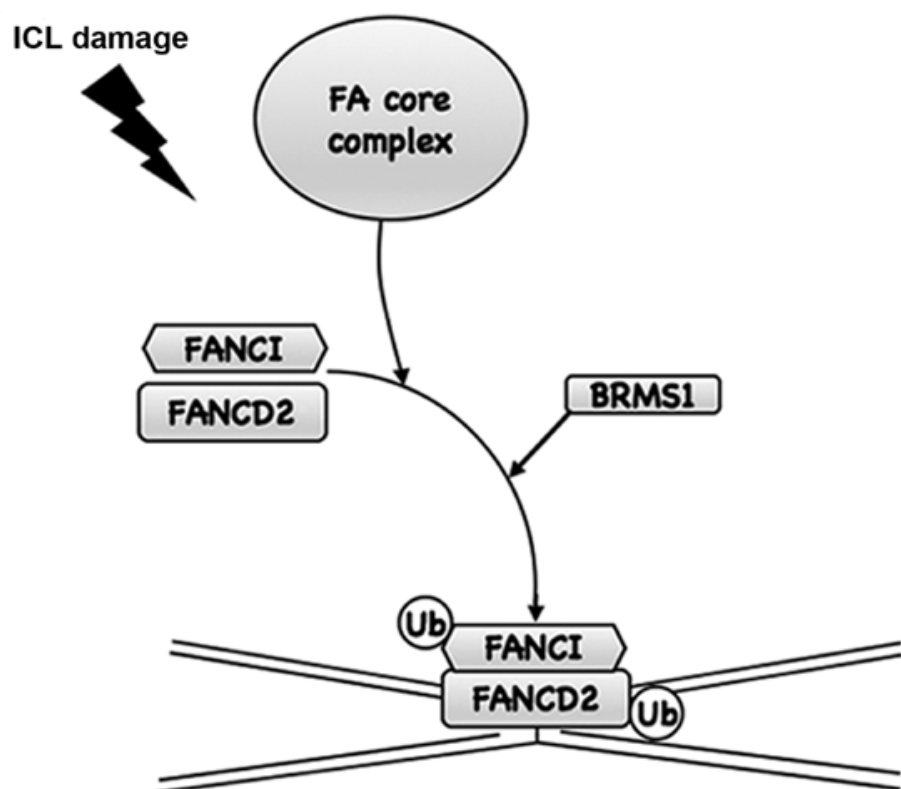

Figure 4. BRMS1-knockout (KO) sensitizes cells to MMC treatment. (A) Clonogenic survival assay of Ctrl and KO cells following different concentrations of MMC treatment. Values are expressed as mean $\pm \mathrm{SD}, \mathrm{n}=5$. (B) Clonogenic survival assay of KO cells reconstituted with the indicated BRMS1 plasmids. Values are expressed as mean $\pm \mathrm{SD}, \mathrm{n}=5$. (C) Graphic representation of the potential role of BRMS1 in the FA pathway. BRMS1 may contribute to the monoubiquitination of FANCI/D2 and FANCD2 localization, and thus promote cell survival upon ICL damage. BRMS1, breast cancer metastasis suppressor 1; MMC, mitomycin C; ICLs, interstrand crosslinks; FA, Fanconi anemia.

in FANCD2 localization upon DNA damage, we further generated a BRMS1-deficient cell clone in the U2OS cell line through CRISPR/Cas9 method as previously described (20). As shown in Fig. 3A, two frame-shift mutations of BRMS1 alleles were introduced into the BRMS1-knockout U2OS clone (KO), leading to complete depletion of BRMS1. As shown in Fig. 3B, while control cells exhibited markedly increased FANCD2 foci $(85.3 \pm 3.9 \%)$ with MMC treatment, BRMS1-deficient cells displayed hypersensitivity to DNA damage, leading to relatively defective FANCD2 foci formation $(59.3 \pm 4.7 \%$ ) (Fig. 3C). This result is consistent with that from the FANCI/D2 monoubiquitination analysis, since modification of FANCI/D2 is pivotal to FANCD2 localization.

To further confirm our finding, a rescue assay was designed. BRMS1-deficient cells were separately reconstituted with full-length BRMS1 (BRMS1-FL) and the BRMS1- $\Delta 3$ mutant without FANCI-interacting ability before being subjected to FANCD2 immunofluorescence staining. As shown in Fig. 3D, FANCD2 signals in the nuclear foci were increased in cells with exogenous BRMS1 expression instead of BRMS1- $\Delta 3$ expression.
Statistically, only the full-length BRMS1, but not the BRMS1- $\Delta 3$ mutant was able to rescue the diminished FANCD2 foci induced by BRMS1 depletion (Fig. 3E). These findings strongly suggest that BRMS1-FANCI interaction may be essential for the regulatory effect of BRMS1 on FANCD2 localization.

BRMS1 contributes to regulation of cell sensitivity to MMC. Based on findings above, we further investigated cell viability upon MMC treatment. As shown in Fig. 4A, U2OS control cells were resistant to high concentrations of $\mathrm{MMC}$, displaying relatively high viability. By contrast, BRMS1-deficient cells (KO) were much more sensitive to MMC in a dose-dependent manner, showing a more than $20 \%$ decrease in the survival ratio with MMC treatment $(120 \mathrm{nM})$. Moreover, cells reconstituted with BRMS1-FL, instead of BRMS1- $\Delta 3$, displayed obvious recovery of cell viability (Fig. 4B). The reduced cell viability in BRMS1-deficient cells not only corresponded to the decrease in FANCD2 foci, but also provided another piece of evidence that BRMS1-FANCI interaction is able to affect the downstream DNA repair process of FA pathway. 


\section{Discussion}

In the present study, FANCI was identified as a novel protein associated with BRMS1 by tandem affinity purification and co-immunoprecipitation. The linker region between two coiled-coil motifs of BRMS1 may be responsible for the interaction, and this domain was also reported to be the binding domain of BRMS1 with other proteins such as p300 and DBC1 $(11,22)$. Further functional studies revealed that depletion of BRMS1 led to decreased FANCI/D2 monoubiquitination, FANCD2 foci formation and cell viability with MMC treatment. Rescue experiments additionally demonstrated that BRMS1-FANCI interaction is necessary for the effect of BRMS1 on the Fanconi anemia (FA) pathway (Fig. 4C). Since proteins reported to interact with BRMS1 are mostly involved in cellular signal transduction and gene expression regulation, our findings bring new insight into the potential function of BRMS1 in genome maintenance.

In the context of the FA pathway, some other proteins have been reported to affect monoubiquitination of the FANCI/D2 complex through protein-protein interaction. For example, RAD18 interacts with FANCD2 and regulates chromatin loading of the FANCI/D2 complex. Depletion of $R A D 18$ reduced the monoubiquitination of FANCI/D2 and finally led to a delay in FANCD2 foci formation together with hypersensitivity of ICL damage (23). In addition, UBL5 could directly interact with FANCI and stabilize FANCI via modulating pre-mRNA splicing of FANCI (24). UBL5 was also important for FANCI/D2 complex formation and monoubiquitination. In our study, while BRMS1 was found to regulate the monoubiquitination of $\mathrm{FANCI} / \mathrm{D} 2$, slight reduction in the FANCI/D2 protein level was also observed in BRMS1-knockdown cells (Fig. 2B). Additional qRT-PCR was carried out and no obvious difference was shown in FANCI/D2 mRNA levels after interference of BRMS1 expression (data not shown). It has been previously shown that FANCI is required for FANCD2 stability, but not vice versa $(18,25,26)$. Moreover, E3 ligase function of BRMS1 induces polyubiquitination of p300 and further proteasome-mediated protein degradation (11), providing another piece of evidence that BRMS1 may affect protein modification and stability of its interacting partners. Whether BRMS1-FANCI interaction may influence the stability of the FANCI/D2 complex remains to be addressed in our future work.

Genome maintenance systems can ensure genome stability via detecting and resolving DNA damages, replication errors, among others. Many tumor-suppressor genes are involved in DNA damage response pathway, since their mutations can facilitate cancer cells to accumulate additional mutations required for transformation. Some of them also contribute to tumor metastasis suppression, such as RAD9, PARP1, BRCA1/2, ATM, TP53, NM23, among others. NM23 is the first identified tumor metastasis suppressor gene (27). The 3'-5'exonuclease activity of NM23 in the DNA repair pathway was demonstrated to be essential for metastasis suppression (28). NM23, instead of its mutant without exonuclease activity, could inhibit invasive capacities of $1205 \mathrm{LU}$ melanoma cells in vitro and suppress spontaneous metastasis in vivo. The potential relationship between the FA pathway and tumor metastasis can also be observed in the well-known tumor suppressors, BRCA1/BRCA2, which are also called FANCS/FANCD1 in the FA pathway (29). They both act in the downstream of the FA pathway. BRCA2 interacts with RAD51 to control its localization and assembly in the DNA damage site, while BRCA1 interacts with the MRE11-RAD50-NBS1 complex implicated in homologous recombination $(30,31)$. Mutations in BRCA1/BRCA2 could decrease the efficiency of the FA pathway and induce genomic instability (27). Moreover, a recent study revealed that the FA/BRCA pathway plays an important role in chemoradiotherapy failure and distant metastasis of cervical cancer (32). In our study, we raised the hypothesis that BRMS1 may be another functional regulator of the FA pathway which is also deeply involved in tumor metastasis. BRMS1 exhibits a strong metastatic suppressive effect in many types of cancers by affecting different steps of the metastatic cascade. Whether BRMS1-FANCI interaction also contributes to the metastatic suppressive role of BRMS1 warrants further investigation.

\section{Acknowledgements}

We thank Professor Jun Huang (Zhejiang University, China) for the kind gift of the pcDNA-FANCI.

\section{Funding}

The present study was supported by the National Key Research and Development Program of China (2017YFC1001101).

\section{Availability of data and materials}

The datasets used and/or analyzed during the current study are available from the corresponding author on reasonable request.

\section{Authors' contributions}

YW, JD, YZ and SQ conceived and designed the experiments. JD and XL performed the co-immunoprecipitation and the western blot analysis. JD and YZ performed RNAi, stable transfectants, immunofluorescence staining and cell viability assay. YW, JD, YZ, XQ, XY and WX participated in the western blot and data analysis. YW, YZ, JD and SQ wrote the paper. All authors read, edited and approved the manuscript and agree to be accountable for all aspects of the research in ensuring that the accuracy or integrity of any part of the work are appropriately investigated and resolved.

\section{Ethics approval and consent to participate}

Not applicable.

\section{Patient consent for publication}

Not applicable.

\section{Competing interests}

The authors declare that they have no competing interests. 


\section{References}

1. Phillips KK, Welch DR, Miele ME, Lee JH, Wei LL and Weissman BE: Suppression of MDA-MB-435 breast carcinoma cell metastasis following the introduction of human chromosome 11. Cancer Res 56: 1222-1227, 1996.

2. Bodenstine TM, Vaidya KS, Ismail A, Beck BH, Cook LM, Diers AR, Landar A and Welch DR: Homotypic gap junctional communication associated with metastasis suppression increases with PKA activity and is unaffected by PI3K inhibition. Cancer Res 70: 10002-10011, 2010

3. Shevde LA, Samant RS, Goldberg SF, Sikaneta T, Alessandrini A, Donahue HJ, Mauger DT and Welch DR: Suppression of human melanoma metastasis by the metastasis suppressor gene, BRMS1. Exp Cell Res 273: 229-239, 2002.

4. Roesley SN, Suryadinata R, Morrish E, Tan AR, Issa SM, Oakhill JS, Bernard O, Welch DR and Śarčević B: Cyclindependent kinase-mediated phosphorylation of breast cancer metastasis suppressor 1 (BRMS1) affects cell migration. Cell Cycle 15: 137-151, 2016.

5. Yang YL, Chen CZ, Jin LP, Ji QQ, Chen YZ, Li Q, Zhang XH and Qu JM: Effect and mechanism of the metastasis suppressor gene BRMS1 on the migration of breast cancer cells. Int J Clin Exp Med 6: 908-916, 2013.

6. Liu Y, Smith PW and Jones DR: Breast cancer metastasis suppressor 1 functions as a corepressor by enhancing histone deacetylase 1-mediated deacetylation of RelA/p65 and promoting apoptosis. Mol Cell Biol 26: 8683-8696, 2006.

7. Wu Y, Jiang W, Wang Y, Wu J, Saiyin H, Qiao X, Mei X, Guo B, Fang X, Zhang L, et al: Breast cancer metastasis suppressor 1 regulates hepatocellular carcinoma cell apoptosis via suppressing osteopontin expression. PLoS One 7: e42976, 2012.

8. Liu Y, Mayo MW, Xiao A, Hall EH, Amin EB, Kadota K, Adusumilli PS and Jones DR: Loss of BRMS1 promotes a mesenchymal phenotype through NF- $\kappa \mathrm{B}$-dependent regulation of Twist1. Mol Cell Biol 35: 303-317, 2015.

9. Meehan WJ, Samant RS, Hopper JE, Carrozza MJ, Shevde LA, Workman JL, Eckert KA, Verderame MF and Welch DR: Breast cancer metastasis suppressor 1 (BRMS1) forms complexes with retinoblastoma-binding protein 1 (RBP1) and the $\mathrm{mSin} 3$ histone deacetylase complex and represses transcription. J Biol Chem 279: 1562-1569, 2004

10. Liu Y, Amin EB, Mayo MW, Chudgar NP, Bucciarelli PR, Kadota K, Adusumilli PS and Jones DR: CK2alpha' drives lung cancer metastasis by targeting BRMS1 nuclear export and degradation. Cancer Res 76: 2675-2686, 2016.

11. Liu Y, Mayo MW, Nagji AS, Hall EH, Shock LS, Xiao A, Stelow EB and Jones DR: BRMS1 suppresses lung cancer metastases through an E3 ligase function on histone acetyltransferase p300. Cancer Res 73: 1308-1317, 2013.

12. Spinola-Amilibia M, Rivera J, Ortiz-Lombardia M, Romero A, Neira JL and Bravo J: The structure of BRMS1 nuclear export signal and SNX6 interacting region reveals a hexamer formed by antiparallel coiled coils. J Mol Biol 411: 1114-1127, 2011.

13. Lobitz S and Velleuer E: Guido fanconi (1892-1979): A jack of all trades. Nat Rev Cancer 6: 893-898, 2006.

14. Auerbach AD: Fanconi anemia and its diagnosis. Mutat Res 668: 4-10, 2009

15. Stoepker C, Ameziane N, van der Lelij P, Kooi IE, Oostra AB, Rooimans MA, van Mil SE, Brink A, Dietrich R, Balk JA, et al: Defects in the Fanconi anemia pathway and chromatid cohesion in head and neck cancer. Cancer Res 75: 3543-3553, 2015.
16. Scharer OD: DNA interstrand crosslinks: Natural and druginduced DNA adducts that induce unique cellular responses. Chembiochem 6: 27-32, 2005.

17. Ceccaldi R, Sarangi P and D'Andrea AD: The Fanconi anaemia pathway: New players and new functions. Nat Rev Mol Cell Biol 17: 337-349, 2016

18. Sims AE, Spiteri E, Sims RJ III, Arita AG, Lach FP, Landers T, Wurm M, Freund M, Neveling K, Hanenberg H, et al: FANCI is a second monoubiquitinated member of the Fanconi anemia pathway. Nat Struct Mol Biol 14: 564-567, 2007.

19. Castella M, Jacquemont C, Thompson EL, Yeo JE, Cheung RS, Huang JW, Sobeck A, Hendrickson EA and Taniguchi T: FANCI regulates recruitment of the FA core complex at sites of DNA damage independently of FANCD2. PLoS Genet 11: e1005563, 2015.

20. Liu X, Ehmed E, Li B, Dou J, Qiao X, Jiang W, Yang X, Qiao S and $\mathrm{Wu} \mathrm{Y}$ : Breast cancer metastasis suppressor 1 modulates SIRT1-dependent p53 deacetylation through interacting with DBC1. Am J Cancer Res 6: 1441-1449, 2016.

21. Livak KJ and Schmittgen TD: Analysis of relative gene expression data using real-time quantitative PCR and the $2^{-\Delta \Delta C_{\mathrm{T}}}$ method. Methods 25: 402-408, 2001.

22. Shimamura A and Alter BP: Pathophysiology and management of inherited bone marrow failure syndromes. Blood Rev 24: 101-122, 2010.

23. Williams SA, Longerich S, Sung P, Vaziri C and Kupfer GM: The E3 ubiquitin ligase RAD18 regulates ubiquitylation and chromatin loading of FANCD2 and FANCI. Blood 117: 5078-5087, 2011.

24. Oka Y, Bekker-Jensen S and Mailand N: Ubiquitin-like protein UBL5 promotes the functional integrity of the Fanconi anemia pathway. EMBO J 34: 1385-1398, 2015.

25. Dorsman JC, Levitus M, Rockx D, Rooimans MA, Oostra AB, Haitjema A, Bakker ST, Steltenpool J, Schuler D, Mohan S, et al: Identification of the Fanconi anemia complementation group I gene, FANCI. Cell Oncol 29: 211-218, 2007.

26. Smogorzewska A, Matsuoka S, Vinciguerra P, McDonald ER III, Hurov KE, Luo J, Ballif BA, Gygi SP, Hofmann K, D'Andrea AD, et al: Identification of the FANCI protein, a monoubiquitinated FANCD2 paralog required for DNA repair. Cell 129: 289-301, 2007.

27. Tutt A, Gabriel A, Bertwistle D, Connor F, Paterson H, Peacock J, Ross G and Ashworth A: Absence of Brca2 causes genome instability by chromosome breakage and loss associated with centrosome amplification. Curr Biol 9: 1107-1110, 1999.

28. Zhang Q, McCorkle JR, Novak M, Yang M and Kaetzel DM: Metastasis suppressor function of NM23-H1 requires its 3'-5'exonuclease activity. Int J Cancer 128: 40-50, 2011.

29. Castro E, Goh C, Olmos D, Saunders E, Leongamornlert D, Tymrakiewicz M, Mahmud N, Dadaev T, Govindasami K, Guy M, et al: Germline BRCA mutations are associated with higher risk of nodal involvement, distant metastasis, and poor survival outcomes in prostate cancer. J Clin Oncol 31: 1748-1757, 2013.

30. Yu DS, Sonoda E, Takeda S, Huang CL, Pellegrini L, Blundell TL and Venkitaraman AR: Dynamic control of Rad51 recombinase by self-association and interaction with BRCA2. Mol Cell 12: 1029-1041, 2003

31. Ree AH, Bratland A, Nome RV, Stokke T and Fodstad $\varnothing$ : Repression of mRNA for the PLK cell cycle gene after DNA damage requires BRCA1. Oncogene 22: 8952-8955, 2003.

32. Balacescu O, Balacescu L, Tudoran O, Todor N, Rus M, Buiga R, Susman S, Fetica B, Pop L, Maja L, et al: Gene expression profiling reveals activation of the FA/BRCA pathway in advanced squamous cervical cancer with intrinsic resistance and therapy failure. BMC Cancer 14: 246, 2014. 